\title{
The enteric bacterial metabolite propionic acid alters brain and plasma phospholipid molecular species: further development of a rodent model of autism spectrum disorders
}

\author{
Raymond H Thomas ${ }^{1 *}$, Melissa M Meeking ${ }^{1}$, Jennifer R Mepham', Lisa Tichenoff', Fred Possmayer², Suya Liu ${ }^{3}$ \\ and Derrick F MacFabe
}

\begin{abstract}
Gastrointestinal symptoms and altered blood phospholipid profiles have been reported in patients with autism spectrum disorders (ASD). Most of the phospholipid analyses have been conducted on the fatty acid composition of isolated phospholipid classes following hydrolysis. A paucity of information exists on how the intact phospholipid molecular species are altered in ASD. We applied ESI/MS to determine how brain and blood intact phospholipid species were altered during the induction of ASD-like behaviors in rats following intraventricular infusions with the enteric bacterial metabolite propionic acid. Animals were infused daily for 8 days, locomotor activity assessed, and animals killed during the induced behaviors. Propionic acid infusions increased locomotor activity. Lipid analysis revealed treatment altered 21 brain and 30 blood phospholipid molecular species. Notable alterations were observed in the composition of brain SM, diacyl mono and polyunsaturated PC, PI, PS, PE, and plasmalogen PC and PE molecular species. These alterations suggest that the propionic acid rat model is a useful tool to study aberrations in lipid metabolism known to affect membrane fluidity, peroxisomal function, gap junction coupling capacity, signaling, and neuroinflammation, all of which may be associated with the pathogenesis of ASD.
\end{abstract}

Keyword: Locomotor activity, Membrane fluidity, Gap junction, Plasmalogens, Docosahexaenoic acid, Oxidative stress

\section{Introduction}

Autism spectrum disorders (ASD) are a family of disorders characterized by stereotypic and restrictive patterns of behavior, deficits in social interactions, and impairments in language development and communication skills [1]. Several recent studies have indicated that interactions between genetic, metabolic, immunological, gastrointestinal, environmental, and behavioral factors may be associated with the pathogenesis of ASD [2-6]. Our research group has been developing a novel rodent model of autism to examine enteric bacterial metabolites as possible environmental triggers in ASD. Such a model could prove useful in investigating how the interactions between the above

\footnotetext{
* Correspondence: rthoma2@uwo.ca

${ }^{1}$ The Kilee Patchell-Evans Autism Research Group, Department of Psychology, University of Western Ontario, London, ON N6A 5C2, Canada

Full list of author information is available at the end of the article
}

factors may contribute to the pathogenesis of ASD [7-12], and identify potential biomarkers that could be used in early detection and screening for this disorder.

The central premise of this model is that propionic acid (PPA) and/or related enteric fatty acids may be candidate environmental factors involved in the development of some types of ASD. Propionate and other short chain fatty acids (for example butyrate, acetate) are produced in the body during normal cellular metabolism and following enteric bacterial fermentation of dietary carbohydrates and proteins [13]. PPA producing enteric bacteria, including unique Clostridial, Desulfovibrio, and Bacteriodetes species, have been isolated from patients with regressive ASD $[2,14]$. Propionate is also present naturally in a variety of foods and is a common food preservative in refined wheat and dairy products [15]. Under normal circumstances these short chain fatty 
acids are primarily metabolized in the liver. However, if there are genetic and/or acquired aberrations in metabolism $[7,16]$, higher than normal levels of short chain fatty acids can be present in the circulating blood, and can cross the gut-blood and blood brain barriers passively and/or actively via high affinity transporters [17]. Under these conditions, short chain fatty acids can concentrate intracellularly, particularly in acidotic conditions $[18,19]$, where they may have deleterious effects on brain development and function [13,20,21]. This could be important in the context of ASD, since PPA is known to affect cell signaling [22], neurotransmitter synthesis and release [20], mitochondrial function/CoA sequestration [16], lipid metabolism [23] immune function [24], gap junction modulation [19], and gene expression [25], all of which have been implicated in ASD [7,25-28].

Previous results from our work developing this model indicate that brief, intracerebroventricular infusions with PPA, but not control compounds (that is, propanol) produced short bouts of behavioral (hyperactivity, perserveration, object fixation, social impairments) and electrophysiological (seizure, caudate spiking) effects, coupled with biochemical (increased oxidative stress, reduced glutathione) and neuropathological (innate neuroinflammation) alterations in adult rats, consistent with those seen in ASD [7-11]. Recent findings with this model indicate infusions with PPA or butyrate altered the brain acylcarnitine and phospholipid profiles [12]. Interestingly, the alterations in rat brain lipids noted in this study showed a number of similarities to those documented in blood from ASD patients [5,29-32].

In the above studies, phospholipid fatty acid composition was determined by analyzing fatty acid acyl groups following hydrolysis of the separated phospholipid classes. Though the standard accepted method of lipid analysis, this method destroys the phospholipid structure, abolishing the ability to establish the individual phospholipid molecular species distribution of the samples. Recently, it has become possible to apply electrospray ionization mass spectrometry to analyze phospholipids as their intact molecular species, allowing for identification of individual and patterns of specific lipids [33-35]. This is potentially important considering there are presently no reliable biomarkers for the detection of ASD, the etiology of ASD is unclear, and altered lipid profiles have been reported both for ASD patients $[5,27,29,32,36]$ and in the PPA rodent model of ASD [12]. Therefore, there is a need to determine: (1) whether or not the intact phospholipid molecular species are altered in relation to behaviors, to further validate this animal model; and (2) how these altered phospholipid profiles may relate to the pathogenesis of ASD.

The development of liquid chromatography electrospray ionization mass spectrometry (LC-ESI/MS) has made it possible to directly analyze phospholipids as intact molecular species, and preserve the information inherent in their chemical structure $[33,35]$. Herein, we used ESI/MS to determine how the intact blood and brain phospholipid molecular species are altered during the induction of abnormal (ASD-like) behaviors following PPA infusions in rats.

\section{Materials and methods Subjects}

Twenty four Long-Evans rats (Charles River Laboratories, Quebec, Canada), weighing 200-225 g (approximately 47-49 days old) at the start of the experiment, were individually housed at $21 \pm 1^{\circ} \mathrm{C}$ in standard acrylic cages $(26 \times 48 \times 21 \mathrm{~cm})$ and exposed to $12: 12 \mathrm{~h}$ light-dark cycle (lights on $0700 \mathrm{~h}$ to $1900 \mathrm{~h}$ ). Animals were allowed access to food (Prolab rat chow) and tap water ad libitum. All procedures were in accordance with guidelines of the Canadian Council on Animal Care and were approved by the University of Western Ontario Animal Use Subcommittee.

\section{Surgical implantation of intracerebroventricular (ICV) cannula}

To induce anesthesia, animals were placed in a sealed Plexiglas box into which $4 \%$ isoflurane and $2 \mathrm{~L} / \mathrm{min}$ oxygen flow were introduced. The animal was then placed into a Kopf stereotaxic frame equipped with a gas flow nose cover to maintain anesthesia throughout surgery at $2 \%$ isoflurane and $500 \mathrm{~mL} / \mathrm{min}$ oxygen flow. Under aseptic conditions, the animal received an implantation of a 23-gauge guide cannula with the tip in the right lateral ventricle (AP $1.3 \mathrm{~mm}$, ML $1.8 \mathrm{~mm}$, DV $3.0 \mathrm{~mm}$ ) [37]. Cannula placements were in accordance with Paxinos and Watson [37] rat brain atlas and our previous experience [7-11]. A removable obturator sealed the guide cannula until an injection was to be made. To facilitate infusion into the lateral ventricle, the tip of a 30-gauge injection cannula protruded $0.5 \mathrm{~mm}$ beyond the tip of the guide cannula. Small stainless steel screws were inserted into the skull surrounding the cannula to provide anchors for dental acrylic, which attached the cannula to the skull. Immediately post-surgery, all rats received a subcutaneous injection of analgesic (Ketoprofin, $1 \mathrm{~mL} / \mathrm{kg}$ ). All animals were allowed 14 days recovery before behavioral testing took place.

\section{Treatment groups and intracerebroventricular infusion procedure}

Following recovery, animals were assigned to one of two groups: PPA treatment $(4.0 \mu \mathrm{L}$ of a $0.26 \mathrm{M}$ solution, $n=12)$, or phosphate buffered saline (PBS) control $(4.0 \mu \mathrm{L}$, $n=12$ ). Sodium propionic acid was dissolved in PBS vehicle and buffered to $\mathrm{pH} 7.5$ using concentrated $\mathrm{HCl}$. Each group received intracerebroventricular (ICV) infusions twice daily, separated by $4 \mathrm{~h}$, for 8 consecutive days. Compounds were infused using a 30-gauge injection cannula 
connected to a Sage syringe pump with sterile PE10 tubing. The tip of the injection cannula protruded $0.5 \mathrm{~mm}$ beyond the tip of the guide cannula. Each injection consisted of 4.0 $\mu \mathrm{L}$ of solution delivered over a period of $1 \mathrm{~min}$. The infusion cannula remained in place for an additional minute before being removed.

\section{Apparatus: automated activity monitors}

Locomotor activity was monitored using three Versamax Animal Activity Monitors (AccuScan Model DCM-8, Columbus, OH, USA). Each monitor consisted of a Plexiglas open field chamber $(40 \times 40 \times 30.5 \mathrm{~cm})$, and a Plexiglas lid with air holes. Located on all four sides of the chamber were 16 infrared beam sensors $2.54 \mathrm{~cm}$ apart and $4.5 \mathrm{~cm}$ from the floor to measure horizontal movements and on two opposite sides were 16 infrared beams located $15 \mathrm{~cm}$ above the chamber floor to measure the vertical movements. Light levels at the floor of each open-field were approximately 900 lux. A VersaMax Analyser (Accuscan Model VSA-16, Columbus, OH, USA) processed relayed data from each automated open-field to a computer located in an adjacent room to the testing chambers $[7,8]$.

\section{Behavioral testing}

The methods used for behavioral assessment presented in this study have been previously reported (2011) [7-12]. Briefly, animals were habituated to the automated activity monitors for two 30-min sessions prior to the treatment sessions. Baseline was recorded on the third day to establish normal activity levels for untreated animals. Locomotor assessments were made for $30 \mathrm{~min}$ immediately following the second ICV infusion daily for 7 days. Locomotor activity was assessed using horizontal, vertical, and repetitive measures (stereotypy) across infusion days. The horizontal activity measures analyzed were number of horizontal movements (the number of horizontal movements separated by $1 \mathrm{~s}$ stop time), horizontal movement time (length of time in seconds an animal was engaged in horizontal movement), and total distance (total horizontal distance $(\mathrm{cm})$ traveled). The vertical activity measure analyzed was number of vertical movements (number of vertical movements separated by $1 \mathrm{~s}$ stop time). The repetitive activity measures were: clockwise revolutions (the number of times an animal ran around in a clockwise circle of at least 2 inches in diameter), counterclockwise revolutions (the number of times an animal ran around in a counterclockwise circle of at least 2 inches in diameter), and the number of stereotypic movements (repeated breaking of the same infrared beam separated by $1 \mathrm{~s}$ or more). Fifteen minutes after animals received the final ICV infusion on the eighth treatment day, they were decapitated, brains quickly removed, placed on powdered dry ice, then transported from surgery room to the analytical laboratory for lipid analysis. Samples were then stored in a $-70^{\circ} \mathrm{C}$ freezer for 2 days before lipid analysis. Whole blood collected from the neck following decapitation was heparinized and spun at $3000 \times \mathrm{g}$ for $5 \mathrm{~min}$, and the supernatant (plasma) collected for lipid analysis.

\section{Lipid extraction}

Brain tissue (100 mg) ipsilateral to ICV infusion site, containing frontal cortex, striatum, thalamus, and dorsal hippocampus, $1.3 \mathrm{~mm}$ posterior to bregma, [37] was ground to fine powder while frozen in liquid nitrogen. The ground brain samples $(100 \mathrm{mg})$ and plasma $(100 \mathrm{uL})$ were transferred to centrifuge tubes containing a mixture $(2.5 \mathrm{~mL})$ of chloroform $/$ methanol/0.01 \% butylated hydroxytoluene (2:1:0.0003; $\mathrm{v} / \mathrm{v} / \mathrm{wt}$ ) and $100 \mu \mathrm{L}$ of $1 \mathrm{mg} / \mathrm{mL}$ 1,2-dipalmitoyl-sn-glycero-3phospho-N,N-dimethylethanolamine (Sigma, St Louis, MO, USA) added as internal standard. The sample and solvent mixture was centrifuged at $10,000 \times \mathrm{g}$ for $15 \mathrm{~min}$. Following centrifugation, the supernatant was collected and $1 \mathrm{~mL}$ of $0.25 \%$ potassium chloride $(\mathrm{KCl})$ was added. The sample was then incubated in a water bath at $70^{\circ} \mathrm{C}$ for $10 \mathrm{~min}$. After incubation, the sample was removed, cooled and the aqueous layer (top) was removed with a Pasteur pipette. The remaining layer (organic) containing the lipids was transferred to pre-weighed $4 \mathrm{~mL}$ sample vial having a poly-teflon resin lined cap (VWR). The sample was dried under a gentle stream of nitrogen and the vial re-weighed to determine the amount of sample recovered [38,39]. The recovered sample was then resuspended in $1 \mathrm{~mL}$ chloroform:methanol $(2: 1 \mathrm{v} / \mathrm{v})$ and stored at $-20^{\circ} \mathrm{C}$ for analysis (ESI/MS).

\section{Electrospray ionization mass spectrometry analysis (ESI-MS)} Aliquots of blood and brain samples were analyzed by infusion on a triple quadruple instrument (Micromass QuattroMicro; Waters, Milford. MA, USA) equipped with a Z-spray source. The mass spectrometer was operated in both the positive and negative ion modes, and precursor ion or neutral loss scans specific to the phospholipid classes were used to determine the composition of the molecular species of each class [33,40]. Precursor ion scan (negative mode) of $\mathrm{m} / \mathrm{z} 153$ was used to detect all glycerophospholipid molecular species, $m / z 241$ used to detect PI molecular species (negative mode), precursor ion scan of $m / z 196$ (negative mode) or neutral loss scan of $141 \mathrm{D}$ (positive mode) used to detect PE molecular species, and neutral loss scan of $87 \mathrm{D}$ (negative mode) or $185 \mathrm{D}$ (positive mode) used to detect PS molecular species; while precursor ion scan of $m / z 184$ in the positive mode was used to detect SM and PC molecular species. The fatty acid composition of the identified molecular species of each phospholipid classes was determined via MS/MS analysis and reference to the literature (LIPID MAPS). The investigated mass range was from $100-1000 \mathrm{~m} / z$. The cone voltage was set at $30 \mathrm{~V}$, the collision energy at $30-50 \mathrm{~V}$, source temperature at $80^{\circ} \mathrm{C}$ and the collision gas at $2 \mathrm{xe}-3$ 
Mbar. Calibration was performed with sodium iodide and mass error was less than 0.05 Dalton (Da). Flow injection was carried out with chloroform:methanol:water:ammonia $(20: 70: 8: 2 \mathrm{v} / \mathrm{v} / \mathrm{v} / \mathrm{v})$ at a flow rate of $50 \mathrm{uL} / \mathrm{min}$ with $20 \mathrm{uL}$ of the sample injected for analysis. Quantitative analysis was conducted using MassLynx 4.0 software (Micromass) and Microsoft Excel by using the signal intensity of the reference standard to normalize the intensities of the identified molecular phospholipid species after correction for ${ }^{13} \mathrm{C}$ isotope effects. Representative external standards for each phospholipid classes (Avanti Polar Lipids, Alabaster, AL, USA) were used to determine the relative amounts of the molecular species within a phospholipid class. The amounts of each molecular species within a phospholipid class are expressed as nanomole percent composition.

\section{Statistical analysis}

A total of 24 animals were used for this study (12 PBS and $12 \mathrm{PPA}$ ).

General analysis of variance (ANOVA) was used to determine the effects of treatments on behavior and phospholipid components. Where treatment effects were significant, the means were compared with Fisher's LSD test, $\alpha=0.05$. A one-way ANOVA was conducted on the baseline data to ensure that there were no individual differences in behavior prior to treatment days. The data were analyzed using the Statistix software package (Analytical Software, Tallahassee, FL, USA).

\section{Results}

\section{Behavioral assessment}

Locomotor behavior was assessed daily for 7 days, immediately following the second infusion of the day. Prior to behavioral assessment, there was no significant difference in baseline behavior between animals assigned to the PPA and PBS treatment groups. Locomotor activity expressed by horizontal, vertical, and repetitive measures across infusion days, was used to assess animal behavior. Analysis of horizontal activity measures revealed a significant effect of PPA treatment across infusion days for number of horizontal movements, horizontal movement time, and total distance traveled (Figure 1). Propionic acid treated animals traveled further, made more horizontal movements, and spent more time traveling horizontally than PBS treated animals. The number of horizontal movements was significantly higher $(F(6,126)=3.69, P<0.001)$ in rats infused with PPA on infusion days $2,4,5,6$, and 7 compared to PBS controls (Figure 1a). A similar pattern was observed for horizontal movement time $(F(6,126)=2.82, P<0.001)$ (Figure 1f) and total distance traveled $(F(6,126)=2.08$, $P<0.001$ ) (Figure $1 \mathrm{~g}$ ), whereby these behavioral activities were significantly higher in PPA treated animals compared to PBS controls on infusion days 2, 3, 4, 5, 6, and 7. Analysis of the number of vertical movements revealed a significant
$(F(6,126)=2.82, P<0.001)$ main effect of PPA treatment across infusion days, indicating PPA treated animals made more vertical movements than PBS treated animals on infusion days 3 through 7 (Figure 1b). The results of the repetitive activity measures showed significant increases in counterclockwise revolutions $(F(6,126)=2.46, P<0.001)$, clockwise revolutions $(F(6,126)=2.40, P<0.001)$ and number of stereotypic movements $(F(6,126)=4.05, P<0.001)$ in PPA-treated animals across infusion days. On infusion days $3,4,5,6$, and 7, animals infused with PPA made more counterclockwise (Figure 1c) and clockwise (Figure 1d) revolutions than PBS controls. Stereotypy showed a similar trend whereby PPA-treated animals displayed more stereotypic movements than PBS animals on infusions days 2 through 7 (Figure 1e).

\section{Lipid analysis}

Behavioral assessment indicated animals developed abnormal (ASD-like) behaviors following PPA infusion (Figure 1). Animals were killed during the expression of these abnormal behaviors on day 8 and the molecular species of five phospholipid classes (SM, PC, PI, PS, and PE) were evaluated to determine whether they were altered during the expression of these behaviors. Phosphatidylcholine and SM molecular species were detected in the positive ion mode (Figure 2), while PI, PS, and PE molecular species were detected in the negative ion mode (Figure 3). Although the same molecular species for each phospholipid class were detected in control and PPA-infused animals, the analyses revealed a quantitative change in these lipid constituents following PPA infusions (Table 1, 2, 3, 4, 5, 6, 7, 8, 9, and 10).

\section{SM molecular species}

Sphingomyelin molecular species containing saturated base (C18:0) and fatty acids predominate in brain samples (Table 1), while species with monounsaturated base (C18:1) and polyunsaturated fatty acid predominate in plasma samples (Table 2).

Propionic acid treatment resulted in alterations in the proportions of both brain $(F(27,56)=10.79, P<0.001)$ and plasma $(F(27,56)=32.64, P<0.001) \mathrm{SM}$ molecular species (Tables 1 and 2). In both brain (Table 1) and plasma (Table 2$)$ an overall increase $(P<0.001)$ was observed in the relative level of saturated species, in addition to a reduction in monounsaturated species. Polyunsaturated species were unchanged in brain, but increased in plasma following PPA infusions. Increased proportions $(P<0.001)$ of $36: 0(18: 0 /$ 18:0) in brain (Table 1) and 38:0 (18:0/20:0) in plasma (Table 2) accounted for the increase in saturated SM species. In brain, the proportions of 38:0 (18:0/20:0) and 40:0 (18:0/ 22:0) were decreased, but this had no overall significant effect on the total brain proportions of saturated SM. Conversely, 38:1 (18:1/20:0) in brain (Table 1) and both 34:1 $(18: 1 / 16: 0)$ and $38: 1 \quad(18: 1 / 20: 0)$ in plasma (Table 2) 


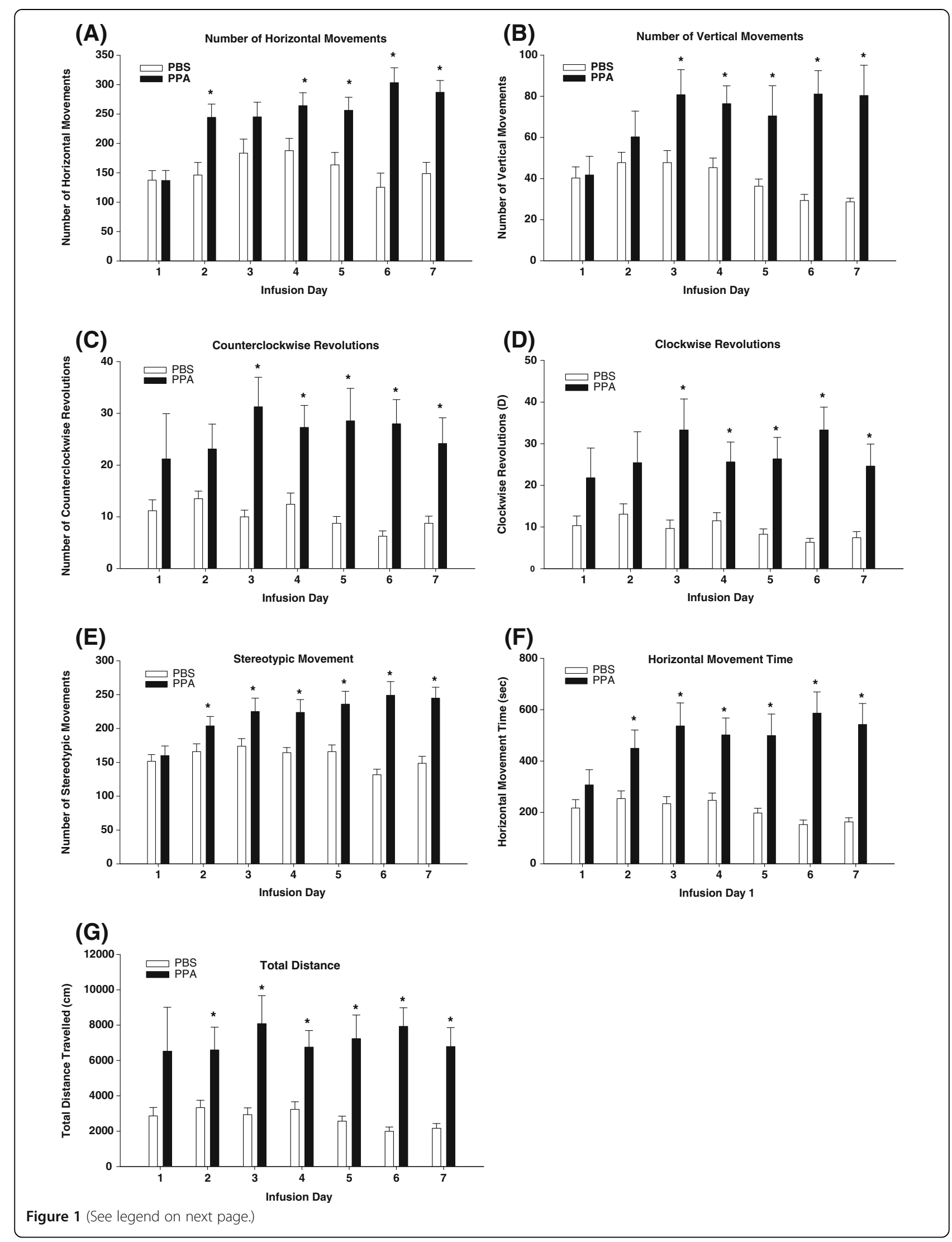


(See figure on previous page.)

Figure 1 Number of horizontal movements (A), number of vertical movements (B), counterclockwise revolutions (C), clockwise revolutions $(D)$, number of stereotypic movements $(E)$, horizontal movement time $(F)$, and total distance travelled $(G)$, representing abnormal animal behavior following ICV infusions with PPA and PBS. Animals received ICV infusions of PPA $(n=12)$ and PBS $(n=12)$ twice a day for 7 consecutive days. Behavioral measurements were monitored immediately after each infusion over 30 min. Values represent means \pm SE over 7 days. Bars accompanied by asterisks indicate significant difference between treatments at $L S D=0.05, n=12$ per treatment group. PBS, phosphate buffered saline solution; PPA, propionic acid.

accounted for the reduction in monounsaturated SM species, while $42: 3(18: 1 / 24: 2)$ in plasma accounted for the increased polyunsaturated species following PPA infusions (Table 2).

\section{PC molecular species}

Diacyl and plasmalogen PC molecular species were present in both brain (Table 3) and plasma (Table 4). However, only the diacyl species were altered $(F$ (59, $120)=1042.12, P<0.001)$ in brain following PPA treatments, while both the diacyl and plasmalogen species were altered $(F(59,120)=873.32, P<0.001)$ in plasma. In brain, the proportions of monounsaturated 34:1 (16:0/18:1) and polyunsaturated diacyl $34: 2(16: 0 / 18: 2)$ and $36: 4$ (16:0/
20:4) species were increased; while a reduction was observed in the monounsaturated diacyl 36:1 (18:0/18:1) species following PPA treatment (Table 3). No overall change was observed in the relative proportions of saturated, unsaturated, and polyunsaturated PC species. Plasma samples on the other hand revealed a reduction in the level of diacyl polyunsaturated PC species (34:2 (16:0/ 18:2), 36:4 (16:0/20:4), 38:2 (18:1/20:1), 38:4 (18:0/20:4)), and an in increase in the proportions of plasmalogen species (36:1 (18:0/18:1)). Although plasma proportions of diacyl saturated 34:0 (16:0/18:0) and monounsaturated 36:1 (18:0/18:1) molecular species increased following PPA infusions, this had no effect on the overall level of total saturated and monounsaturated PC species (Table 4).

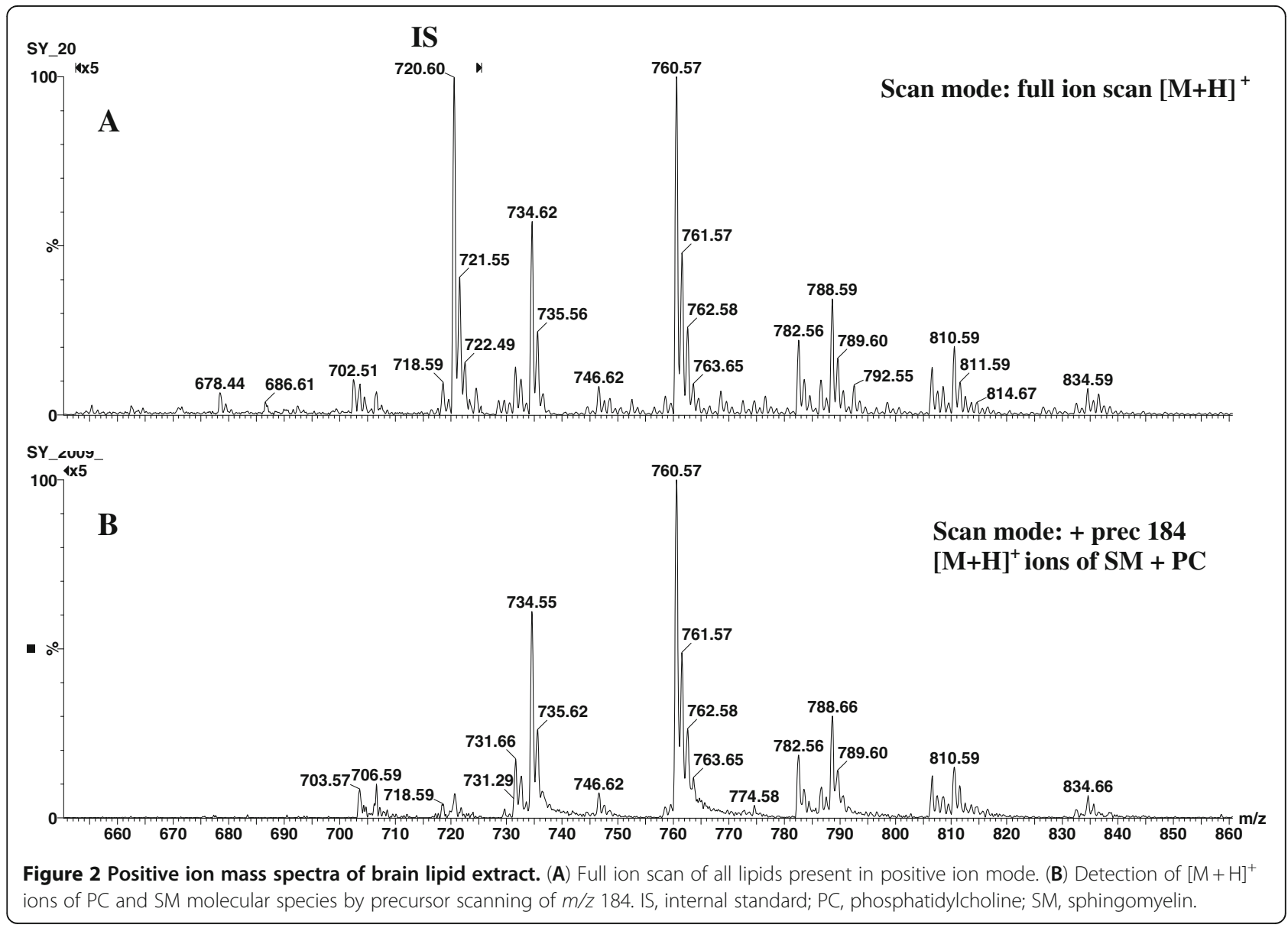




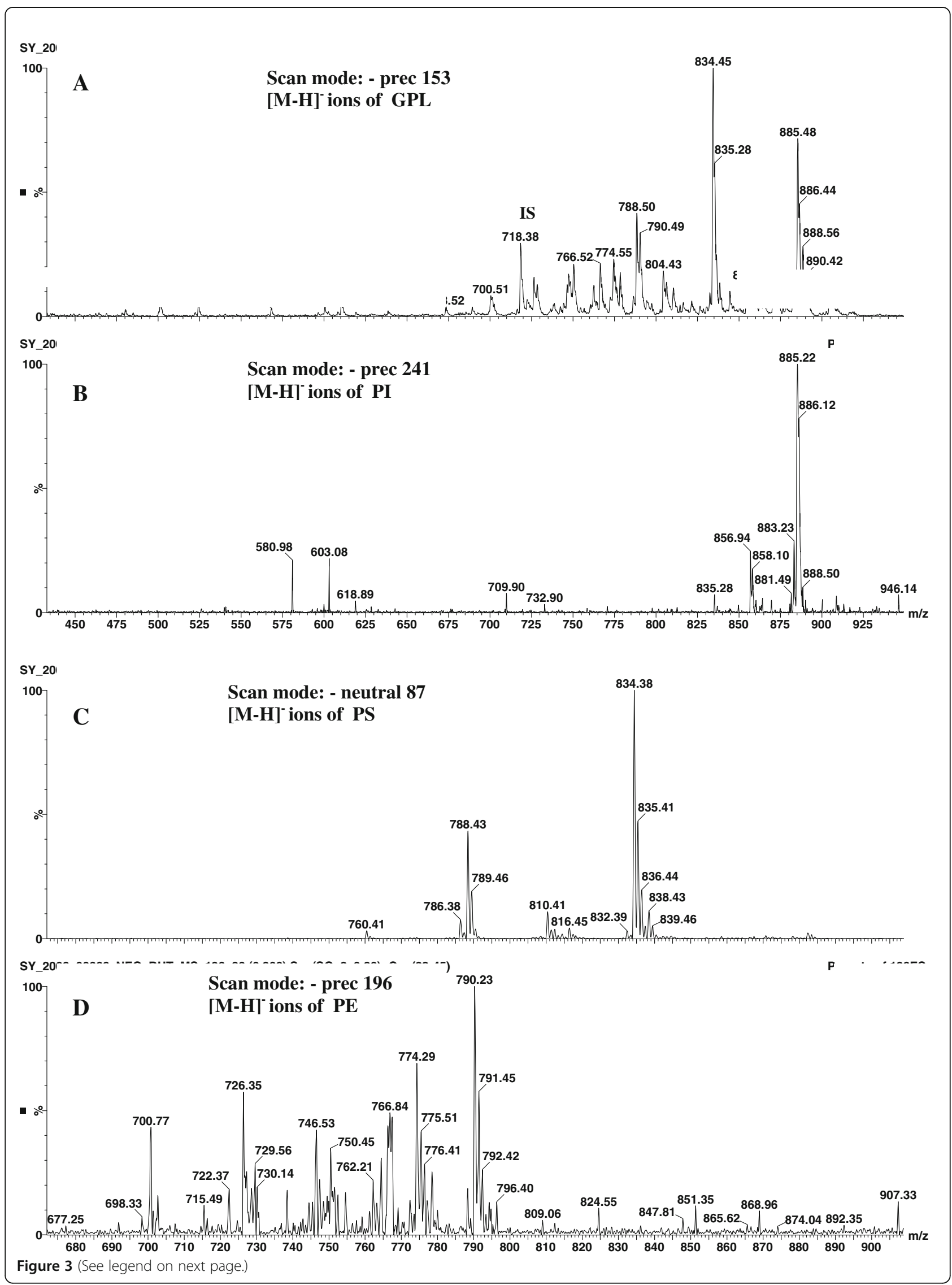


(See figure on previous page.)

Figure 3 Negative ion mass spectra of brain lipid extract. (A) Detection of all $[\mathrm{M}-\mathrm{H}]{ }^{-}$ions of glycerophospholipids by precursor ion scan of $\mathrm{m} / \mathrm{z}$ 153. (B) Detection of PI molecular species by precursor ion scanning of $m / z 241$. (C) Detection of PS molecular species by neutrol loss of 87 D. (D) Detection of PE molecular species by precursor ion scanning of $\mathrm{m} / z$ 196. GPL, glycerophospholipids; IS, internal standard; PI, phosphatidylinositol; PS, phosphatidylserine; PE, phosphatidylethanolamine.

\section{PI molecular species}

Propionic acid treatment altered the proportions of several plasma $(F(35,72)=75.31, P<0.001)$ PI molecular species, while the proportions of only two brain $(F(35$, $72)=2017.70, \quad P<0.001) \quad$ PI molecular species were affected (slightly reduced) (Table 5 and 6). Reduced proportions of polyunsaturated diacyl $36: 3$ (18:1/18:2) and 40:4 (18:0/22:4) species were observed in brain samples following PPA treatment (Table 5). The alterations were more varied with plasma PI molecular species. The proportions of plasma diacyl 34:1 (16:0/18:1), $36: 3$ (18:1/ 18:2), 38:6 (16:0/22:6), 40:4 (18:0/22:4), and 40:6 (18:0/ $22: 6)$ increased, while the proportions of the diacyl $38: 4$ (18:0/20:4) species decreased following PPA treatment (Table 6). These changes indicate a compositional shift occurred particularly in plasma PI molecular species following PPA infusions.

\section{PS molecular species}

Propionic acid infusions altered the proportions of both mono and polyunsaturated PS species in brain (Table 7)

\begin{tabular}{|c|c|c|c|c|}
\hline $\begin{array}{l}\text { Molecular } \\
\text { weight }\end{array}$ & $\begin{array}{l}\text { Molecular } \\
\text { species }\end{array}$ & $\begin{array}{l}\text { Base/acyl } \\
\text { species }\end{array}$ & PBS & PPA \\
\hline 703 & $34: 1$ & $18: 1 / 16: 0$ & $0.07 \pm 0.03$ & $0.10 \pm 0.05$ \\
\hline 733 & $36: 0$ & 18:0/18:0 & $21.96 \pm 0.35$ & $39.13 \pm 1.35^{*}$ \\
\hline 731 & $36: 1$ & 18:1/18:0 & $6.21 \pm 0.67$ & $4.53 \pm 1.28$ \\
\hline 761 & $38: 0$ & 18:0/20:0 & $38.09 \pm 0.84$ & $29.37 \pm 1.92^{*}$ \\
\hline 759 & $38: 1$ & 18:1/20:0 & $16.08 \pm 0.19$ & $12.31 \pm 3.64^{*}$ \\
\hline 789 & $40: 0$ & 18:0/22:0 & $13.03 \pm 0.24$ & $9.92 \pm 1.31^{*}$ \\
\hline 811 & $42: 3$ & $18: 1 / 24: 2$ & $4.56 \pm 0.12$ & $4.64 \pm 1.54$ \\
\hline Total\% & & & 100 & 100 \\
\hline$\sum$ Saturates & & & $73.07 \pm 0.15$ & $78.41 \pm 1.44^{*}$ \\
\hline$\sum$ Monounsat & & & $22.37 \pm 0.66$ & $16.94 \pm 2.91 *$ \\
\hline$\sum$ Polyunsat & & & $4.55 \pm 0.12$ & $4.63 \pm 1.54$ \\
\hline
\end{tabular}

Values (nanomole percent by weight composition) represent means \pm standard errors. Means in the same row accompanied by asterisks are significantly different between treatments at $\mathrm{LSD}=0.05, n=12$ per treatment group. Monounsat, monounsaturated fatty acids; PBS, phosphate buffered saline solution; PPA, propionic acid; polyunsat, polyunsaturated fatty acids. Sphingomyelin molecular species were identified using a precursor ion scan of $\mathrm{m} / \mathrm{z} 184$ in ESI positive mode. The lipid components in the table are arranged based on the molecular species composition. and plasma (Table 8$)$. In both brain $(F(21,44)=305.49$, $P<0.001)$ and plasma $(F(21,44)=12.14, P<0.001)$, an overall increase was observed in the proportion of monounsaturated species, while a reduction was observed in polyunsaturated species. An increase in brain 36:1 (18:0/ 18:1) (Table 7) and plasma (Table 8) 34:1 (16:0/18:1) and $36: 1$ (18:0/18:1) accounted for the increase in monounsaturated PS species. Conversely, a reduction in brain proportions of 40:4 (18:0/22:4) and 40:6 (18:0/22:6) and plasma proportions of 40:3 (18:0/22:3) and 40:6 (18:0/ 22:6) accounted for the reduced polyunsaturated PS species following PPA infusions.

\section{PE molecular species}

Compositional changes in brain diacyl and plasmalogen PE species occurred following PPA infusions (Table 9). Propionic acid treatment reduced $(F(79,160)=8.79$, $P<0.001) P<0.001)$ brain relative proportions of plasmalogen 40:6 (p18:0/22:6), diacyl polyunsaturated 38:6 (16:0/ $22: 6)$, and 40:6 (18:0/22:6) species, but increased the proportions of plasmalogen 36:1 (p18:0/18:1), 36:2 (p18:0/

Table 2 Changes (percent composition) in rat plasma sphingomyelin molecular species following intraventricular infusion with propionic acid (PPA) and phosphate buffered saline (PBS)

\begin{tabular}{lllll}
\hline $\begin{array}{l}\text { Molecular } \\
\text { weight }\end{array}$ & $\begin{array}{l}\text { Molecular } \\
\text { species }\end{array}$ & $\begin{array}{l}\text { Base/acyl } \\
\text { species }\end{array}$ & PBS & PPA \\
\hline 703 & $34: 1$ & $18: 1 / 16: 0$ & $14.05 \pm 1.42$ & $4.63 \pm 1.10^{*}$ \\
733 & $36: 0$ & $18: 0 / 18: 0$ & $2.63 \pm 0.47$ & $3.40 \pm 1.23$ \\
731 & $36: 1$ & $18: 1 / 18: 0$ & $1.54 \pm 0.32$ & $1.65 \pm 0.42$ \\
761 & $38: 0$ & $18: 0 / 20: 0$ & $12.91 \pm 0.37$ & $15.92 \pm 0.65^{*}$ \\
759 & $38: 1$ & $18: 1 / 20: 0$ & $8.36 \pm 0.56$ & $5.77 \pm 0.24^{*}$ \\
789 & $40: 0$ & $18: 0 / 22: 0$ & $9.44 \pm 2.89$ & $10.39 \pm 2.03$ \\
811 & $42: 3$ & $18: 1 / 24: 2$ & $51.07 \pm 0.62$ & $58.24 \pm 0.68^{*}$ \\
Total\% & & & $\mathbf{1 0 0}$ & $\mathbf{1 0 0}$ \\
$\sum$ Saturates & & & $\mathbf{2 4 . 9 8} \pm \mathbf{1 . 3 3}$ & $\mathbf{2 9 . 7 2} \pm \mathbf{1 . 4 9 *}$ \\
$\sum$ Monounsat & & & $\mathbf{2 3 . 9 5} \pm \mathbf{1 . 5 3}$ & $\mathbf{1 2 . 0 5} \pm \mathbf{1 . 5 7 *}$ \\
$\sum$ Polyunsat & & & $\mathbf{5 1 . 0 6} \pm \mathbf{0 . 6 3}$ & $\mathbf{5 8 . 2 2} \pm \mathbf{0 . 6 8 *}$ \\
\hline
\end{tabular}

Values (nanomole percent by weight composition) represent means \pm standard errors. Means in the same row accompanied by asterisks are significantly different between treatments at $\mathrm{LSD}=0.05, n=12$ per treatment group. Monounsat, monounsaturated fatty acids; PBS, phosphate buffered saline solution; PPA, propionic acid; polyunsat, polyunsaturated fatty acids. Sphingomyelin molecular species were identified using a precursor ion scan of $m / z 184$ in ESI positive mode. The lipid components in the table are arranged based on the molecular species composition. 
Table 3 Changes (percent composition) in rat brain phosphatidylcholine molecular species following intraventricular infusion with propionic acid (PPA) and phosphate buffered saline (PBS)

\begin{tabular}{|c|c|c|c|c|}
\hline Molecular weight & Molecular species & Plasmalogen or diacyl species & PBS & PPA \\
\hline 706 & D30:0 & $16: 0 / 14: 0$ & $0.13 \pm 0.055$ & $0.13 \pm 0.04$ \\
\hline 734 & D32:0 & $16: 0 / 16: 0$ & $19.23 \pm 0.34$ & $19.33 \pm 0.69$ \\
\hline 718 & P32:1 & p16:0/16:1 & $0.05 \pm 0.01$ & $0.06 \pm 0.01$ \\
\hline 762 & D34:0 & $16: 0 / 18: 0$ & $18.66 \pm 0.49$ & $19.14 \pm 0.63$ \\
\hline 760 & D34:1 & 16:0/18:1 & $32.80 \pm 0.15$ & $33.68 \pm 0.16^{*}$ \\
\hline 746 & P34:1 & p16:0/18:1 & $1.54 \pm 0.26$ & $1.40 \pm 0.32$ \\
\hline 758 & D34:2 & 16:0/18:2 & $0.27 \pm 0.04$ & $0.69 \pm 0.19^{*}$ \\
\hline 788 & D36:1 & 18:0/18:1 & $10.53 \pm 0.18$ & $9.20 \pm 0.15^{*}$ \\
\hline 774 & P36:1 & p18:0/18:1 & $0.50 \pm 0.11$ & $0.40 \pm 0.11$ \\
\hline 782 & D36:4 & $16: 0 / 20: 4$ & $3.96 \pm 0.51$ & $4.81 \pm 0.53^{*}$ \\
\hline 814 & D38:2 & 18:1/20:1 & $1.21 \pm 0.25$ & $1.29 \pm 0.11$ \\
\hline 812 & D38:3 & 18:0/20:3 & $1.85 \pm 0.28$ & $1.74 \pm 0.14$ \\
\hline 810 & D38:4 & 18:0/20:4 & $4.91 \pm 0.26$ & $4.33 \pm 0.34$ \\
\hline 806 & D38:6 & 16:0/22:6 & $2.98 \pm 0.12$ & $2.74 \pm 0.10$ \\
\hline 834 & D40:6 & $18: 0 / 22: 6$ & $1.36 \pm 0.11$ & $1.06 \pm 0.14$ \\
\hline Total\% & & & 100 & 100 \\
\hline$\sum$ Saturates & & & $18.78 \pm 0.48$ & $19.27 \pm 0.60$ \\
\hline$\sum$ Monounsat & & & $62.56 \pm 0.51$ & $62.20 \pm 0.70$ \\
\hline$\sum$ Polyunsat & & & $16.55 \pm 0.63$ & $16.65 \pm 0.99$ \\
\hline$\sum$ Plas & & & $2.09 \pm 0.32$ & $1.85 \pm 0.43$ \\
\hline
\end{tabular}

Values (nanomole percent by weight composition) represent means \pm standard errors. Means in the same row accompanied by asterisks are significantly different between treatments at $L S D=0.05, n=12$ per treatment group. $\mathrm{D}$ represents diacyl species and $\mathrm{P}$ represents plasmalogens species, $\mathrm{p}$ denotes a sn-1 vinyl ether (plasmalogen) linkage. Monounsat, monounsaturated fatty acids; PBS, phosphate buffered saline solution; plas, plasmalogens; polyunsat, polyunsaturated fatty acids; PPA, propionic acid. Phosphatidylcholine molecular species were identified using a precursor ion scan of $\mathrm{m} / \mathrm{z} 184$ in ESI positive mode.

18:2), 38:4 (p18:0/20:4), and 40:4 (p18:0/22:4) molecular species. In plasma, both the composition and overall relative proportions of $\mathrm{PE}$ diacyl and plasmalogen species were altered following PPA infusions (Table 10). A reduction $(F(79,160)=11.61, P<0.001)$ was observed in diacyl 36:1 (18:0/18:1), plasmalogen 36:0 (p18:0/18:0), 36:1 (p18:0/18:1), 36:4 (p16:0/20:4), 38:4 (p18:0/20:4), and 40:6 (p18:0/22:6) molecular species. In contrast, the proportions of polyunsaturated diacyl species (38:4 (18:0/20:4) and 38:6 (16:0/22:6)) increased with PPA infusions.

\section{Discussion}

\section{Behavioral assessment}

Locomotor activity as expressed by horizontal, vertical, and repetitive measures across infusion days was used to assess abnormal animal behavior. Assessment of locomotor activity indicates that PPA infusions induced hyperactivity and stereotypy in rodents consistent with the findings from previous studies by our group [7-12]. These behaviors bear some resemblance to the hyperactive and repetitive behaviors which are recognized as core symptoms of ASD [41]. PPA and other short chain fatty acids are known to increase intracellular neuronal and glial acidification and calcium proportions, thereby producing widespread effects on neurotransmitter release including glutamate, dopamine, norepinepherine, and serotonin, each of which play a role in elicitation of locomotor activity, as seen in the present study [42-44]. In addition, PPA has been shown to increase glutamatergic transmission, leading to excitability in brain regions linked to locomotor activity $[45,46]$. Collectively, these observations are consistent with an emerging theory of ASD as a disruption of excitatory/ inhibitory neuronal activity [47]. The fact that PPA infusions consistently induce elevations in locomotor activity in rodents indicates that this model may be a useful tool for studying the neurological mechanisms involved in the behavioral disturbances seen in ASD.

\section{Alterations in phospholipid molecular species}

The phospholipid profiles observed in control animals are consistent with those reported in the literature for rat brain $([48,49]$ with the exception of SM molecular species. Typically, molecular species with sphingosine base (C18:1) make up the largest proportion of the SM profile in mammals. In this study, molecular species with sphinganine bases (C18:0) accounted for the largest proportion of rat brain 
Table 4 Changes (percent composition) in rat plasma phosphatidylcholine molecular species following intraventricular infusion with propionic acid (PPA) and phosphate buffered saline (PBS)

\begin{tabular}{|c|c|c|c|c|}
\hline Molecular weight & Molecular species & Plasmalogen or diacyl species & PBS & PPA \\
\hline 706 & D30:0 & 16:0/14:0 & $0.79 \pm 0.42$ & $0.59 \pm 0.18$ \\
\hline 734 & D32:0 & 16:0/16:0 & $1.39 \pm 0.57$ & $2.08 \pm 0.14$ \\
\hline 718 & P32:1 & p16:0/16:1 & $0.52 \pm 0.39$ & $0.40 \pm 0.053$ \\
\hline 762 & D34:0 & 16:0/18:0 & $2.90 \pm 0.34$ & $3.80 \pm 0.32^{*}$ \\
\hline 760 & D34:1 & 16:0/18:1 & $9.03 \pm 0.73$ & $9.98 \pm 1.81$ \\
\hline 746 & P34:1 & p16:0/18:1 & $0.61 \pm 0.28$ & $0.52 \pm 0.05$ \\
\hline 758 & D34:2 & 16:0/18:2 & $20.13 \pm 0.23$ & $17.61 \pm 0.16^{*}$ \\
\hline 788 & D36:1 & 18:0/18:1 & $8.11 \pm 0.40$ & $9.87 \pm 0.38^{*}$ \\
\hline 774 & P36:1 & $\mathrm{p} 18: 0 / 18: 1$ & $0.76 \pm 0.24$ & $6.24 \pm 1.96^{*}$ \\
\hline 782 & D36:4 & $16: 0 / 20: 4$ & $13.23 \pm 0.42$ & $11.41 \pm 0.22^{*}$ \\
\hline 814 & D38:2 & $18: 1 / 20: 1$ & $4.14 \pm 0.24$ & $2.97 \pm 0.45^{*}$ \\
\hline 812 & D38:3 & 18:0/20:3 & $9.82 \pm 2.05$ & $8.70 \pm 0.64$ \\
\hline 810 & D38:4 & 18:0/20:4 & $23.92 \pm 0.41$ & $21.45 \pm 0.87^{*}$ \\
\hline 806 & D38:6 & 16:0/22:6 & $2.42 \pm 0.64$ & $2.60 \pm 0.63$ \\
\hline 834 & D40:6 & 18:0/22:6 & $2.23 \pm 0.68$ & $1.78 \pm 0.58$ \\
\hline Total\% & & & 100 & 100 \\
\hline$\sum$ Saturates & & & $3.68 \pm 0.58$ & $4.39 \pm 1.21$ \\
\hline$\sum$ Monounsat & & & $18.52 \pm 1.57$ & $21.92 \pm 2.25$ \\
\hline$\sum$ Polyunsat & & & $75.89 \pm 2.55$ & $66.52 \pm 2.09 *$ \\
\hline$\sum$ Plas & & & $1.89 \pm 0.86$ & $7.15 \pm 1.84^{*}$ \\
\hline
\end{tabular}

Values (nanomole percent by weight composition) represent means \pm standard errors. Means in the same row accompanied by asterisks are significantly different between treatments at $L S D=0.05, n=12$ per treatment group. $D$ represents diacyl species and $P$ represents plasmalogens species, $p$ denotes a sn- 1 vinyl ether (plasmalogen) linkage., Monounsat, monounsaturated fatty acids; PBS, phosphate buffered saline solution; plas, plasmalogens; polyunsat, polyunsaturated fatty acids; PPA, propionic acid. Phosphatidylcholine molecular species were identified using a precursor ion scan of $\mathrm{m} / \mathrm{z} 184$ in ESI positive mode. The lipid components in the table are arranged based on the molecular species composition.

Table 5 Changes (percent composition) in rat brain phosphatidylinositol molecular species following intraventricular infusion with propionic acid (PPA) and phosphate buffered saline (PBS)

\begin{tabular}{lllll}
\hline Molecular weight & Molecular species & Diacyl species & PBS & PPA \\
\hline 835 & D34:1 & $16: 0 / 18: 1$ & $0.53 \pm 0.05$ & $0.54 \pm 0.07$ \\
859 & D36:3 & $18: 1 / 18: 2$ & $1.01 \pm 0.14$ & $0.79 \pm 0.01^{*}$ \\
857 & D36:4 & $16: 0 / 20: 4$ & $7.71 \pm 0.32$ & $7.02 \pm 0.45$ \\
887 & D38:3 & $18: 0 / 20: 3$ & $9.07 \pm 0.69$ & $9.71 \pm 0.73$ \\
885 & D38:4 & $18: 0 / 20: 4$ & $74.17 \pm 1.35$ & $74.60 \pm 1.27$ \\
883 & D38:5 & $18: 1 / 20: 4$ & $5.53 \pm 0.73$ & $5.44 \pm 0.41$ \\
881 & D38:6 & $16: 0 / 22: 6$ & $0.80 \pm 0.04$ & $0.79 \pm 0.04$ \\
913 & D40:4 & $18: 0 / 22: 4$ & $0.41 \pm 0.01$ & $0.17 \pm 0.03^{*}$ \\
909 & D40:6 & $18: 0 / 22: 6$ & $0.77 \pm 0.10$ & $\mathbf{0}$ \\
Total\% & & & $\mathbf{1 0 0}$ & $\mathbf{1 0 0}$ \\
$\sum$ Monounsat & & & $\mathbf{0 . 5 3} \pm \mathbf{0 . 0 5}$ & $\mathbf{0 . 5 4} \pm \mathbf{0 . 0 7}$ \\
$\sum$ Polyunsat & & & $\mathbf{9 9 . 4 6 \pm 0 . 0 5}$ & $\mathbf{9 9 . 4 6 \pm 0 . 0 7}$
\end{tabular}

Values (nanomole percent by weight composition) represent means \pm standard errors. Means in the same row accompanied by asterisks are significantly different between treatments at $L S D=0.05, n=12$ per treatment group. D represents diacyl species. Monounsat, monounsaturated fatty acids; PBS, phosphate buffered saline solution, polyunsat, polyunsaturated fatty acids; PPA, propionic acid. Phosphatidylinositol molecular species were identified using a precursor ion scan of $m /$ $z 241$ in ESI negative mode. The lipid components in the table are arranged based on the molecular species composition. 
Table 6 Changes (percent composition) in rat plasma phosphatidylinositol molecular species following intraventricular infusion with propionic acid (PPA) and phosphate buffered saline (PBS)

\begin{tabular}{|c|c|c|c|c|}
\hline Molecular weight & Molecular species & Diacyl/species & PBS & PPA \\
\hline 835 & D34:1 & 16:0/18:1 & $6.71 \pm 0.66$ & $9.04 \pm 0.39^{*}$ \\
\hline 859 & D36:3 & 18:1/18:2 & $6.16 \pm 0.29$ & $8.67 \pm 0.97^{*}$ \\
\hline 857 & D36:4 & $16: 0 / 20: 4$ & $8.33 \pm 1.03$ & $8.70 \pm 0.85$ \\
\hline 887 & D38:3 & 18:0/20:3 & $8.53 \pm 0.63$ & $7.72 \pm 0.69$ \\
\hline 885 & D38:4 & 18:0/20:4 & $44.00 \pm 2.19$ & $32.68 \pm 3.6^{*}$ \\
\hline 883 & D38:5 & $18: 1 / 20: 4$ & $6.67 \pm 0.56$ & $7.79 \pm 0.28$ \\
\hline 881 & D38:6 & $16: 0 / 22: 6$ & $6.17 \pm 0.04$ & $7.89 \pm 0.50^{*}$ \\
\hline 913 & D40:4 & $18: 0 / 22: 4$ & $6.50 \pm 0.60$ & $8.47 \pm 0.31^{*}$ \\
\hline 909 & D40:6 & 18:0/22:6 & $6.93 \pm 0.54$ & $9.04 \pm 0.38^{*}$ \\
\hline Total\% & & & 100 & 100 \\
\hline$\sum$ Monounsat & & & $6.70 \pm 0.66$ & $9.03 \pm 1.03$ \\
\hline$\sum$ Polyunsat & & & $93.29 \pm 0.66$ & $90.96 \pm 1.03$ \\
\hline
\end{tabular}

Values (nanomole percent by weight composition) represent means \pm standard errors. Means in the same row accompanied by asterisks are significantly different between treatments at $L S D=0.05, n=12$ per treatment group. D represents diacyl species. Monounsat, monounsaturated fatty acids; PBS, phosphate buffered saline solution, polyunsat, polyunsaturated fatty acids; PPA, propionic acid. Phosphatidylinositol molecular species were identified using a precursor ion scan of $\mathrm{m} / \mathrm{z} 241$ in ESI negative mode. The lipid components in the table are arranged based on the molecular species composition.

SM profile. At this time, we have no suitable/conclusive explanation for this observed variation in brain SM profile following careful perusal of the data to ensure mass spectral interpretation, data normalization, and calculations are correct to the best of our knowledge. However, a similar SM profile with saturated base predominating has been reported previously in human retina [40].
Phospholipids are the major structural components of neuronal and other cellular membranes, and include PC, PE, PS, PI, and SM [50,51]. All of these phospholipid classes were observed to have altered molecular species distribution following PPA infusion. It has been suggested that common neurodevelopmental disorders such as ASD could be associated with functional deficiencies or imbalances in fatty acid

Table 7 Changes (nanomole percent by weight composition) in rat brain phosphatidylserine molecular species following intraventricular infusion with propionic acid (PPA) and phosphate buffered saline (PBS)

\begin{tabular}{|c|c|c|c|c|}
\hline Molecular weight & Molecular species & Diacyl species & PBS & PPA \\
\hline 760 & D34:1 & 16:0/18:1 & $0.90 \pm 0.10$ & $1.10 \pm 0.21$ \\
\hline 790 & D36:0 & 18:0/18:0 & $1.59 \pm 0.41$ & $1.65 \pm 0.11$ \\
\hline 788 & D36:1 & 18:0/18:1 & $18.10 \pm 0.53$ & $21.62 \pm 0.63^{*}$ \\
\hline 786 & D36:2 & 18:0/18:2 & $2.36 \pm 0.34$ & $2.55 \pm 0.17$ \\
\hline 816 & D38:1 & 18:0/20:1 & $1.00 \pm 0.15$ & $1.22 \pm 0.19$ \\
\hline 810 & D38:4 & 18:0/20:4 & $4.18 \pm 0.07$ & $4.46 \pm 0.12$ \\
\hline 840 & D40:3 & 18:0/22:3 & $1.19 \pm 0.23$ & $1.20 \pm 0.17$ \\
\hline 838 & D40:4 & 18:0/22:4 & $4.67 \pm 0.17$ & $3.63 \pm 0.14^{*}$ \\
\hline 836 & D40:5 & 18:0/22:5 & $6.40 \pm 0.30$ & $6.91 \pm 0.43$ \\
\hline 834 & D40:6 & 18:0/22:6 & $58.39 \pm 0.98$ & $54.49 \pm 1.05^{*}$ \\
\hline 832 & D40:7 & 18:1/22:6 & $1.22 \pm 0.08$ & $1.17 \pm 0.12$ \\
\hline Total\% & & & 100 & 100 \\
\hline$\sum$ Saturates & & & $1.59 \pm 0.15$ & $1.65 \pm 0.11$ \\
\hline$\sum$ Monounsat & & & $19.99 \pm 0.39$ & $23.93 \pm 0.66^{*}$ \\
\hline$\sum$ Polyunsat & & & $78.41 \pm 0.58$ & $74.45 \pm 0.55^{*}$ \\
\hline
\end{tabular}

Values (nanomole percent by weight composition) represent means \pm standard errors. Means in the same row accompanied by asterisks are significantly different between treatments at $\mathrm{LSD}=0.05, n=12$ per treatment group. D represents diacyl species. Monounsat, monounsaturated fatty acids; PBS, phosphate buffered saline solution; polyunsat, polyunsaturated fatty acids; PPA, propionic acid. Phosphatidylserine molecular species were identified using neutral loss scan of $87 \mathrm{D}$ in ESI negative mode. The lipid components in the table are arranged based on the molecular species composition. 
Table 8 Changes (nanomole percent by weight composition) in rat plasma phosphatidylserine molecular species following intraventricular infusion with propionic acid (PPA) and phosphate buffered saline (PBS)

\begin{tabular}{|c|c|c|c|c|}
\hline Molecular weight & Molecular species & Diacyl species & PBS & PPA \\
\hline 762 & D34:1 & $16: 0 / 18: 1$ & $6.55 \pm 0.30$ & $7.94 \pm 0.09^{*}$ \\
\hline 792 & D36:0 & 18:0/18:0 & $8.12 \pm 0.57$ & $7.62 \pm 0.24$ \\
\hline 790 & D36:1 & 18:0/18:1 & $9.53 \pm 0.74$ & $12.13 \pm 1.21^{*}$ \\
\hline 788 & D36:2 & 18:0/18:2 & $7.81 \pm 0.06$ & $8.84 \pm 0.63^{*}$ \\
\hline 818 & D38:1 & 18:0/20:1 & $7.72 \pm 0.24$ & $6.94 \pm 0.63$ \\
\hline 812 & D38:4 & 18:0/20:4 & $11.55 \pm 1.36$ & $11.76 \pm 1.65$ \\
\hline 842 & D40:3 & 18:0/22:3 & $8.64 \pm 0.53$ & $7.37 \pm 0.51^{*}$ \\
\hline 840 & D40:4 & 18:0/22:4 & $7.81 \pm 0.08$ & $7.50 \pm 0.35$ \\
\hline 838 & D40:5 & 18:0/22:5 & $7.61 \pm 0.23$ & $7.69 \pm 0.06$ \\
\hline 836 & D40:6 & 18:0/22:6 & $16.67 \pm 1.34$ & $13.68 \pm 0.88^{*}$ \\
\hline 834 & D40:7 & 18:1/22:6 & $7.99 \pm 0.37$ & $8.53 \pm 0.19$ \\
\hline Total\% & & & 100 & 100 \\
\hline$\sum$ Saturates & & & $8.12 \pm 0.57$ & $7.62 \pm 0.24$ \\
\hline$\sum$ Monounsat & & & $23.80 \pm 1.76$ & $27.02 \pm 1.99^{*}$ \\
\hline$\sum$ Polyunsat & & & $68.06 \pm 0.99$ & $65.34 \pm 1.18^{*}$ \\
\hline
\end{tabular}

Values (nanomole percent by weight composition) represent means \pm standard errors. Means in the same row accompanied by asterisks are significantly different between treatments at $\mathrm{LSD}=0.05, n=12$ per treatment group. D represents diacyl species. Monounsat, monounsaturated fatty acids; PBS, phosphate buffered saline solution; polyunsat, polyunsaturated fatty acids; PPA, propionic acid. Phosphatidylserine molecular species were identified using neutral loss scan of $185 \mathrm{D}$ in ESI positive mode. The lipid components in the table are arranged based on the molecular species composition.

synthesis/metabolism $[5,27,29,36]$. Most of these former studies evaluated the fatty acid composition following hydrolysis of either total or individual phospholipid classes. Alterations in specific phospholipid molecular species could not only contribute more specific clinical criteria, but could also provide a basis for mechanistic interpretations. However, to date, only one study [27] has evaluated the intact phospholipid molecular species in the blood of ASD patients. Further, these authors only analyzed PE phospholipid molecular species. In the present study, we evaluated $\mathrm{SM}, \mathrm{PC}, \mathrm{PE}, \mathrm{PS}$, and PI molecular species following infusion with PPA and the induction of abnormal (ASD-like) behaviors. Alterations were observed in 21 brain and 30 blood phospholipid molecular species. Pastural et al. [27] observed elevations in the relative proportions of plasmalogen $\mathrm{PE}$, saturated, and polyunsaturated PE molecular species in the plasma of ASD patients. In our plasma analyses we also observed elevations in the relative proportions of polyunsaturated PE species, but the relative reduction in plasmalogen PE was in contrast with their findings. In addition, plasma from PPA-infused rats demonstrated elevations in the relative proportions of some saturated SM and PC molecular species, diacyl and plasmalogen monounsaturated PC species, PS monounsaturated species, and some polyunsaturated PI molecular species (36:3, 40:4, and 40:6). Elevations were also observed in the proportions of some brain PC polyunsaturated molecular species, PS monounsaturated, and PE plasmalogen species in the present study.
Analysis of SM, PC, PI, PE, PS, and PC phospholipid classes were conducted with both brain and blood samples. In many cases, the same phospholipid molecular species were altered in both blood and brain, but the direction and the relative proportions of these alterations were not consistent between both sample types. Much of the published work demonstrating alterations in lipid metabolism in autism has been done using plasma obtained from ASD patients $[5,27,32,36]$. The findings from these studies include elevations in saturated fatty acids $[27,29,30]$, accompanied by a decline in plasmalogens $[5,29,30]$, mono and polyunsaturated $(\omega 3+\omega 6)$ fatty acids [5,29,30,32,36,52]. Conversely, others have reported elevations in the proportions of mono and poly unsaturated fatty acids $[5,27,30,53]$. However, the origin of these altered fatty acids and plasmalogens are unknown, because the structures of the phospholipids were destroyed by hydrolysis during sample analysis. This paper is the most comprehensive study to date which examines intact PL molecular species alteration in relation to autism. Currently, there is no clear uniform mechanism governing the etiology and early detection of ASD, and no accepted biomarkers are available. Although this study is descriptive in nature, it provides considerably more information than is currently available, and as such provides a foundation for defining which intact phospholipid molecular species can be altered in relation to ASD. This could provide a framework for future studies to elucidate the mechanisms 
Table 9 Changes (percent composition) in rat brain phosphatidylethanolamine molecular species following intraventricular infusion with propionic acid (PPA) and phosphate buffered saline (PBS)

\begin{tabular}{|c|c|c|c|c|}
\hline Molecular weight & Molecular species & Plasmalogen or diacyl species & PBS & PPA \\
\hline 700 & P34:1 & p16:0/18:1 & $5.85 \pm 0.15$ & $5.10 \pm 0.14^{*}$ \\
\hline 714 & D34:2 & 16:0/18:2 & $3.37 \pm 0.17$ & $3.49 \pm 0.15$ \\
\hline 730 & P36:0 & p18:0/18:0 & $3.42 \pm 0.13$ & $4.03 \pm 0.38$ \\
\hline 744 & D36:1 & 18:0/18:1 & $3.50 \pm 0.46$ & $3.32 \pm 0.37$ \\
\hline 728 & P36:1 & p18:0/18:1 & $6.00 \pm 0.09$ & $6.82 \pm 0.12^{*}$ \\
\hline 742 & D36:2 & 18:0/18:2 & $3.68 \pm 0.14$ & $3.59 \pm 0.35$ \\
\hline 726 & P36:2 & p18:0/18:2 & $4.79 \pm 0.30$ & $7.16 \pm 0.62^{*}$ \\
\hline 724 & P36:3 & p18:1/18:2 & $3.38 \pm 0.42$ & $2.98 \pm 0.27$ \\
\hline 722 & P36:4 & p16:0/20:4 & $4.32 \pm 0.12$ & $4.43 \pm 0.14$ \\
\hline 766 & D38:4 & 18:0/20:4 & $6.99 \pm 0.71$ & $7.33 \pm 0.62$ \\
\hline 750 & P38:4 & p18:0/20:4 & $6.07 \pm 0.13$ & $7.70 \pm 0.02^{*}$ \\
\hline 762 & D38:6 & $16: 0 / 22: 6$ & $4.18 \pm 0.16$ & $3.45 \pm 0.15^{*}$ \\
\hline 746 & P38:6 & p16:0/22:6 & $5.14 \pm 0.45$ & $5.11 \pm 0.80$ \\
\hline 796 & D40:3 & 18:0/22:3 & $3.59 \pm 0.26$ & $3.52 \pm 0.43$ \\
\hline 794 & D40:4 & 20:0/20:4 & $3.51 \pm 0.08$ & $3.85 \pm 0.42$ \\
\hline 778 & P40:4 & p18:0/22:4 & $3.46 \pm 0.26$ & $5.00 \pm 0.10^{*}$ \\
\hline 792 & D40:5 & 18:0/22:5 & $4.30 \pm 0.31$ & $3.93 \pm 0.41$ \\
\hline 776 & P40:5 & p18:0/22:5 & $3.91 \pm 0.69$ & $3.79 \pm 0.60$ \\
\hline 790 & D40:6 & 18:0/22:6 & $11.07 \pm 1.06$ & $8.59 \pm 0.36^{*}$ \\
\hline 774 & P40:6 & p18:0/22:6 & $9.47 \pm 0.21$ & $6.82 \pm 0.86^{*}$ \\
\hline Total\% & & & 100 & 100 \\
\hline$\sum$ Saturates & & & $3.42 \pm 0.12$ & $4.02 \pm 0.38$ \\
\hline$\sum$ Monounsat & & & $15.32 \pm 0.65$ & $15.23 \pm 0.92$ \\
\hline$\sum$ Polyunsat & & & $81.23 \pm 0.69$ & $80.73 \pm 0.55$ \\
\hline$\sum$ Plas & & & $55.82 \pm 1.63$ & $58.93 \pm 1.40$ \\
\hline
\end{tabular}

Values (nanomole percent by weight composition) represent means \pm standard errors. Means in the same row accompanied by asterisks are significantly different between treatments at $\mathrm{LSD}=0.05, n=12$ per treatment group. $\mathrm{D}$ represents diacyl species and $\mathrm{P}$ represents plasmalogens species, $\mathrm{p}$ denotes a sn-1 vinyl ether (plasmalogen) linkage. Monounsat, monounsaturated fatty acids; PBS, phosphate buffered saline solution; plas, plasmalogens; polyunsat, polyunsaturated fatty acids; PPA, propionic acid. Phosphatidylethanolamine molecular species were identified using a precursor ion scan of $\mathrm{m} / z$ 196 in ESI negative mode. The lipid components in the table are arranged based on the molecular species composition.

associated with the observed lipid alterations and their relationship to behavioral changes in ASD.

The use of an animal model allowed comparison of brain and plasma PL during the period of PPA-induced ASD-like behaviors. The observations that small amounts (1.04 micromole/infusion) of PPA into brain can influence plasma lipid composition are considered intriguing. However, the plasma alterations noted did not correlate directly with those in brain. It could be that this difference merely reflects the nature of the PPA rodent model where treatment is limited to 8 days. Furthermore, ASD likely involves genetic, metabolic, and environmental factors which could result in systemic as well as CNS effects.

It is evident from the data presented in this study that PPA infusion produced small but significant alterations in the composition of brain and plasma phospholipid species.
These alterations occurred independent of diet. The most notable alterations were observed in the composition of brain SM, diacyl mono and polyunsaturated PC, PI, PS, PE, and plasmalogen $\mathrm{PC}$ and $\mathrm{PE}$ molecular species. These observations are considered interesting because alterations in brain lipid composition, particularly during development can potentially have serious consequences on CNS function.

\section{Potential physiological consequences of altered} phospholipid molecular species and their relation to ASD Lipid mediated signaling and neuroinflammation in ASD

The pathological consequences of disturbances in phospholipid metabolism could include alterations in signal transduction involving the generation of second messengers derived from docosahexaenoic (C22:6n3) and arachidonic (C20:4n6) acids [54]. The observation that PPA infusion 
Table 10 Changes (nanomole percent by weight composition) in rat plasma phosphatidylethanolamine molecular species following intraventricular infusion with propionic acid (PPA) and phosphate buffered saline (PBS)

\begin{tabular}{|c|c|c|c|c|}
\hline Molecular weight & Molecular species & Plasmalogen or diacyl species & PBS & PPA \\
\hline 702 & P34:1 & p16:0/18:1 & $4.59 \pm 0.43$ & $3.51 \pm 0.53$ \\
\hline 716 & D34:2 & 16:0/18:2 & $4.42 \pm 0.13$ & $4.41 \pm 0.66$ \\
\hline 732 & P36:0 & p18:0/18:0 & $4.39 \pm 0.19$ & $3.11 \pm 0.41^{*}$ \\
\hline 746 & D36:1 & 18:0/18:1 & $4.55 \pm 0.44$ & $3.38 \pm 0.49^{*}$ \\
\hline 730 & P36:1 & p18:0/18:1 & $5.76 \pm 0.56$ & $3.57 \pm 0.50 *$ \\
\hline 744 & D36:2 & 18:0/18:2 & $5.20 \pm 0.58$ & $4.49 \pm 0.55$ \\
\hline 728 & P36:2 & p18:0/18:2 & $3.10 \pm 1.36$ & $3.19 \pm 0.26$ \\
\hline 726 & P36:3 & p18:1/18:2 & $4.28 \pm 0.19$ & $3.85 \pm 0.36$ \\
\hline 724 & P36:4 & p16:0/20:4 & $4.47 \pm 0.42$ & $3.41 \pm 0.46^{*}$ \\
\hline 768 & D38:4 & 18:0/20:4 & $10.82 \pm 0.71$ & $23.60 \pm 4.93^{*}$ \\
\hline 752 & P38:4 & p18:0/20:4 & $4.67 \pm 0.06$ & $3.70 \pm 0.45^{*}$ \\
\hline 764 & D38:6 & $16: 0 / 22: 6$ & $5.35 \pm 0.21$ & $7.02 \pm 0.56^{*}$ \\
\hline 748 & P38:6 & p16:0/22:6 & $4.87 \pm 0.38$ & $3.71 \pm 0.64$ \\
\hline 798 & D40:3 & 18:0/22:3 & $4.27 \pm 0.34$ & $3.92 \pm 0.68$ \\
\hline 796 & D40:4 & 20:0/20:4 & $4.78 \pm 0.29$ & $3.67 \pm 0.62$ \\
\hline 780 & P40:4 & p18:0/22:4 & $4.06 \pm 0.17$ & $3.41 \pm 0.34$ \\
\hline 794 & D40:5 & 18:0/22:5 & $4.97 \pm 0.35$ & $4.07 \pm 0.32$ \\
\hline 778 & P40:5 & p18:0/22:5 & $4.67 \pm 0.27$ & $3.76 \pm 0.83$ \\
\hline 792 & D40:6 & 18:0/22:6 & $5.89 \pm 0.62$ & $7.03 \pm 1.63$ \\
\hline 776 & P40:6 & p18:0/22:6 & $4.80 \pm 0.33$ & $3.54 \pm 0.69^{*}$ \\
\hline Total\% & & & 100 & 100 \\
\hline$\sum$ Saturates & & & $4.39 \pm 0.19$ & $3.11 \pm 0.41^{*}$ \\
\hline$\sum$ Monounsat & & & $14.92 \pm 0.39$ & $10.46 \pm 1.46^{*}$ \\
\hline$\sum$ Polyunsat & & & $80.68 \pm 0.43$ & $86.41 \pm 1.81 *$ \\
\hline$\sum$ Plas & & & $49.69 \pm 0.96$ & $38.37 \pm 3.81 *$ \\
\hline
\end{tabular}

Values (nanomole percent by weight composition) represent means \pm standard errors. Means in the same row accompanied by asterisks are significantly different between treatments at $\mathrm{LSD}=0.05, n=12$ per treatment group. $\mathrm{D}$ represents diacyl species and $\mathrm{P}$ represents plasmalogens species, $\mathrm{p}$ denotes a sn-1 vinyl ether (plasmalogen) linkage. Monounsat, monounsaturated fatty acids; PBS, phosphate buffered saline solution, plas, plasmalogens; polyunsat, polyunsaturated fatty acids; PPA, propionic acid. Phosphatidylethanolamine molecular species were identified using neutral loss scan of $141 \mathrm{D}$ in ESI positive mode. The lipid components in the table are arranged based on the molecular species composition.

increased the proportions of brain PI and PC molecular species containing arachidonic acid and decreased the proportions of PS and PE molecular species with docosahexaenoic acid, suggests that PPA could influence the innate neuroinflammatory process observed in autism. Metabolism of arachidonic and docosahexaenoic acids released from the sn-2 position of the glycerol moiety by phospholipase A2 results in the formation of eicosanoids and docosanoids, respectively [54]. Both eicosanoids and docosanoids are potent modulators of the inflammatory response system. Eicosanoids are inflammatory mediators that induce the formation of proinflammatory cytokines such as tumor necrosis factor (TNF), interleukin 1 (IL-1), and interleukin 6 (IL-6). Elevated levels of TNF, IL-1, and IL-6 have been reported in plasma of ASD patients [28]. Docosanoids on the other hand are antiflammatory and include protectins and resolvins that have known neuroprotective effects [54]. There is now emerging evidence that autism may be accompanied by abnormalities in the inflammatory response system [28], and that this abnormality may be related to the increases in oxidative stress [55-57], innate neuroinflammation [58], and altered lipid profiles $[5,27,30,32,53]$ reported in ASD.

The increased accumulation of brain molecular species with the eicosanoids precursor (arachidonic acid), and the reduced proportions of the molecular species containing the docosanoids precursor (docosahexaenoic acid) observed in this study are interesting, considering the increased innate neuroinflammation (reactive astrogliosis and activated microglia) previously observed with this model [7] and reported for the brain of ASD patients at autopsy [58].

Several of the studies analyzing the hydrolyzed fatty acid components obtained from the blood of ASD patients also report alterations in the proportions of arachidonic and 
docosahexaenoic acids [27,29,32,36]. This could indicate aberrations in fatty acid elongation and desaturation may occur in the etiology of ASD. Arachidonic and docosahexaenoic acids are cleaved from the sn-2 position of the glycerol moiety by phospholipase A2. Genetic sites linked to autism on chromosome $8 \mathrm{q} 22$ are in the proximity of the gene (8q24) for secretory phospholipase A2 [36]. Collectively, these findings suggest possible involvements of arachidonate, docosahexaenoate, and phospholipase A2 in the signal cascade associated with the innate neuroinflammation observed in ASD.

\section{Membrane fluidity and stability}

Biological membranes are predominantly bilayers in which the inner and outer leaflets have different phospholipid compositions. In contrast, blood plasma phospholipids are present as monolayers, sourrounding lipoproteins, and the effect of fluidity on lipoprotein function is not well understood. Here we will refer to both cellular bilayers and blood lipoprotein monolayers collectively as 'membranes'. Phospholipid molecular species distribution influences bilayer physical properties such as fluidity and this affects membrane protein function. Monolayer molecular species composition also affects physical properties. Organisms can adjust the order or fluidity of their cellular membranes in response to changes in their physiological environment by altering their lipid composition [50]. Alterations in brain and blood phospholipid composition consistent with an adjustment in the order or fluidity of the membrane in these tissues in response to PPA infusions were observed in this study. This adjustment was reflected by changes in the relative proportions of unsaturated, diacyl, and/or plasmalogen species. Alterations in desaturation can have profound changes on membrane fluidity because increased carboncarbon double bonds make unsaturated fatty acids more mobile, flexible, and fluid [50]. Changes in the relative proportions and the composition of diacyl and plasmalogen forms of phospholipids can also impact the fluidity of the membrane. For example, plasmalogen species facilitate membrane fusion six times faster than diacyl species [59].

Phosphatidylcholine, which has a large polar head group does not pack closely in membrane bilayers and tends to be more fluid compared to PE, which has a small head group and packs more closely in membranes, making them less fluid at physiological temperatures. The PC/PE balance in cell membranes is thought to regulate membrane fluidity and stabilize the membrane $[50,60]$. In both brain and blood membranes, PPA infusions alter the PC and PE composition (both diacyl and plasmalogen forms) possibly disrupting this balance. Alterations in brain membrane lipid composition affecting fluidity have been found to be associated with a number of behavioral abnormalities, as well as neurological and psychiatric disorders in both adults and children [61]. Alterations in membrane fluidity affect membrane properties, which in turn can affect the functions of integral membrane proteins, ion channels and the permeability of solutes across the membrane [54]. It is unclear at this preliminary stage whether or not any of these processes are affected by PPA infusion in this model, or if they are related to the etiology of ASD. These are the subjects of further studies in our laboratory.

\section{Lipid oxidation/peroxisomal function}

Plasmalogens are vinyl ether lipids found in PE, PC and PS. Several studies have reported increases [27] or declines $[5,29,30]$ in plasmalogens obtain from the bloods of ASD patients. Typically plasmalogens have docosahexaenoic or arachidonic acids esterified in the sn-2 position of the glycerol moiety, and are essential for normal brain development and functions. Reduced plasmalogens and docosahexaenoic acid levels are characteristic of peroxisomal-associated neurological disorders such as infantile Refsum disease, adrenoleukodystrophy, adrenomyeloneuropathy, and Zellweger's syndrome $[5,27,59]$. The only study we know that analyzed the intact PE molecular species of blood obtained from ASD patients observed an overall increase in PE plasmalogens species, and this increase was accompanied by an increase in docosahexaenoic acid containing molecular species [27]. In our rat model, we observed an overall reduction in the relative proportions of PE plasmalogens in plasma, inclusive of species with docosahexaenoic acid at the sn- 2 position of the glycerol moiety. However, consistent with [27], elevated proportions of PE polyunsaturated diacyl species were observed in plasma, inclusive of docosahexaenoate containing species.

In brain, a reduction in the relative proportions of diacyl and plasmalogen PE molecular species containing docosahexaenoic acid was observed in this study following PPA infusion. This was accompanied by a relative increase in several other PE plasmalogen molecular species lacking docosahexaenoic acid in the sn-2 position of the glycerol moiety. Plasmalogens act as a reservoir for docosahexaenoic acid, and both compounds have synthetic steps that occur in the peroxisome, providing a biochemical link [5]. Peroxisomal disorders are characterized by abnormal peroxisomal biogenesis associated with altered functionality of the two rate limiting enzymes in plasmalogen synthesis, acyl-coenzyme A (CoA): dihydroxyacetonephosphate acyltransferase and alkyldihydoxyacetonephosphate synthase $[36,59]$. It appears from the findings presented in this study and those from previous reports $[5,27,29,30,52]$ that aberrations in plasmalogen metabolism may occur in ASD, implying that peroxisomal dysfunction could be involved.

Plasmalogens are considered endogenous antioxidants, because their vinyl ether bonds are efficient neutralizers of reactive oxygen species, which damage the polyunsaturated fatty acids present in the sn-2 position of plasmalogen phospholipids [62]. The alterations observed in plasmalogen molecular species in relation to the alterations in brain and 
blood polyunsaturated PE and PC molecular species, may be a response to increase oxidation following PPA infusions. This is very interesting because oxidative damage of lipids has been suggested to play a part in the pathogenesis of many neurological diseases including autism $[26,55]$. In addition, increased oxidative stress, and decreased antioxidant capacity have been previously reported in this rodent model $[7,8]$, and also found to be present in ASD patients [55-57]. Interestingly, oxidative damage has been shown to uncouple the gap junctions in astrocytes [63,64]. Arachidonic and docosahexaenoic acids which are very susceptible to oxidative damage have been shown to modulate the coupling capacity of gap junctions [65-67]. In this study, PPA infusion led to alterations in the proportions of brain arachidonic and docosahexaenoic acids containing molecular species.

Collectively, the findings presented in this study, along with those observed in the blood of ASD patients; indicate that alterations in peroxisomal associated lipid metabolism and increased oxidative stress, possibly predisposing polyunsaturated fatty acids to oxidative damage may be associated with the pathogenesis of ASD.

\section{Conclusion}

Infusions with PPA-induced abnormal (ASD-like) behaviors in rodents consistent with previous studies. This induction in abnormal behaviors was accompanied by alterations in several brain and blood SM, PC, PS, PE, and PI molecular species. Alterations in lipid composition are known to affect membrane fluidity, peroxisomal functions, gap junction coupling capacity, and signaling during neuroinflammation, which may be associated with the pathogenesis of ASD, at least in a subset of patients. The mechanisms governing these findings and their potential relevance to the pathophysiology of ASD, particularly during development, is the subject of further studies in our laboratory. Finally, these PPA-induced alterations of brain and plasma phospholipid molecular species provide further validation of this rodent model as a useful tool to link the disparate behavioral, central nervous system, and systemic findings with a plausible environmental factor in ASD.

\section{Abbreviations \\ ASD: Autism spectrum disorder; BUT: Butyric acid; CNS: Central nervous system; ESI: MS electrospray ionization mass spectrometry; ICV: Intracerebroventricular; PBS: Phosphate buffered saline; PC: Phosphatidylcholine; PE: Phosphatidylethanolamine; PI: Phosphatidylinositol; PPA: Propionic acid; PS: Phosphatidylserine; PUFA: Polyunsaturated fatty acids; SM: Sphingomyelin.}

\section{Competing interests}

The authors declare no conflict of interest.

\section{Authors' contributions}

RHT designed and conducted the experiments for the lipid component of the study and wrote the manuscript. MMM designed and conducted the experiments for the behavioral component of the study and assisted in editing the manuscript. JRM assisted with lipid and data analysis and editing the manuscript. LT assisted with surgery and editing the manuscript. FP cosupervised the study, assisted with interpretations and editing the manuscript. SL assisted with electrospray analysis and editing the manuscript. DFM supervised the study and assisted with editing the manuscript. All authors discussed the results/ implications, commented on the manuscript at all stages; read and approved the final manuscript.

\section{Acknowledgements}

The authors would like to thank Miss Lisa Huyhn, for her assistance with data entry. The authors would also like to thank Dr Christopher Guglielmo

(Department of Biology, UWO) for his contribution to data interpretation and the use of his laboratory space and equipment. This research was supported by contributions from Goodlife Children's Foundation and Autism Research Institute Foundation to Derrick MacFabe. We extend our heartfelt gratitude to David Patchell-Evans and Kilee Patchell-Evans.

\section{Author details}

${ }^{1}$ The Kilee Patchell-Evans Autism Research Group, Department of Psychology, University of Western Ontario, London, ON N6A 5C2, Canada. ${ }^{2}$ Department of Obstetrics/Gynecology and Biochemistry, University of Western Ontario, London Health Sciences Center, London, ON, Canada. ${ }^{3}$ Biological Mass Spectrometry Laboratory, Department of Biochemistry, University of Western Ontario, London, ON, Canada.

Received: 8 February 2012 Accepted: 29 May 2012

Published: 2 July 2012

\section{References}

1. Amminger GP, Berger GE, Schafer MR, Klier C, Friedrich MH, Feucht M: Omega-3 fatty acids supplementation in children with autism: a double-blind randomized, placebo-controlled pilot study. Biol Psychiatry 2007, 61:551-553.

2. Finegold SM, Molitoris D, Song Y, Liu C, Vaisanen ML, Bolte E, McTeague M, Sandler R, Wexler H, Marlowe EM, Collins MD, Lawson PA, Summanen P, Baysallar M, Tomzynski TJ, Read E, Johnson E, Rolfe R, Nasir P, Shah H, Haake DA, Manning P, Kaul A: Gastrointestinal microflora studies in late-onset autism. Clin Infect Dis 2002, 35:S6-S16.

3. Hu WW, Frank BC, Heine S, Lee NH, Quackenbush J: Gene expression profiling of lymphoblastoid cell lines from monozygotic twins discordant in severity of autism reveals differential regulation of neurologically relevant genes. BMC Genomics 2006, 7:118.

4. Sarachana T, Zhou R, Chen G, Manji HK, Hu WW: Investigation of post-transcriptional gene regulatory networks associated with autism spectrum disorders by microRNA expression profiling of lymphoblastoid cell lines. Genome Med 2010, 2:23.

5. Wiest MM, German JB, Harvey DJ, Watkins SM, Hertz-Picciotto I: Plasma fatty acid profiles in autism: a case-control study. Prostaglandins Leukot Essent Fatty Acids 2009, 80:221-227.

6. Zerrate MC, Pletnikov M, Connors SL, Vargas DL, Seidler FJ, Zimmerman AW, Slotkin TA, Pardo CA: Neuroinflammation and behavioral abnormalities after neonatal terbutaline treatment in rats: implications for autism. $J$ Pharmacol Exp Ther 2007, 322:16-22.

7. MacFabe DF, Cain DP, Rodriguez-Capote K, Franklin AE, Hoffman JE, Boon F, Taylor AR, Kavaliers M, Ossenkopp KP: Neurobiological effects of intraventricular propionic acid in rats: possible role of short chain fatty acids on the pathogenesis and characteristics of autism spectrum disorders. Behav Brain Res 2007, 176:149-169.

8. MacFabe DF, Rodriguez-Capote K, Hoffman JE, Franklin AE, Mohammad-Asef $Y$, Taylor A, Boon F, Cain DP, Kavaliers M, Possmayer F, Ossenkopp KP: A novel rodent model of autism: Intraventricular infusions of propionic acid increase locomotor activity and induce neuroinflammation and oxidative stress in discrete regions of adult rat brain. Am J Biochem \& Biotech. 2008, 4:146-166.

9. MacFabe DF, Cain NE, Boon F, Ossenkopp KP, Cain DP: Effects of the enteric bacterial metabolic product propionic acid on object-directed behavior, social behavior, cognition, and neuroinflammation in adolescent rats: Relevance to autism spectrum disorder. Behav Brain Res 2010, 217:47-54

10. Shultz SR, MacFabe DF, Martin S, Jackson J, Taylor R, Boon F, Ossenkopp KP, Cain DP: Intracerebroventricular injections of the enteric bacterial metabolic product propionic acid impair cognition and sensorimotor 
ability in the Long-Evans rat: further development of a rodent model of autism. Behav Brain Res 2009, 200:33-41.

11. Shultz SR, MacFabe DF, Ossenkopp KP, Scratch S, Whelan J, Taylor R, Cain DP: Intracerebroventricular injection of propionic acid, an enteric bacterial metabolic end-product, impairs social behavior in the rat: implications for an animal model of autism. Neuropharmacology 2008, 54:901-911.

12. Thomas RH, Foley KA, Mepham JR, Tichenoff LJ, Possmayer F, MacFabe DF: Altered brain phospholipid and acylcarnitine profiles in propionic acid infused rodents: further development of a potential model of autism spectrum disorders. J Neurochem 2010, 113:515-529.

13. Mortensen PB, Clausen MR: Short-chain fatty acids in the human colon: relation to gastrointestinal health and disease. Scand J Gastroenterol Suppl 1996, 216:132-148.

14. Finegold SM, Dowd SE, Gontcharova V, Liu C, Henley KE, Wolcott RD, Youn E, Summanen PH, Granpeesheh D, Dixon D, Liu M, Molitoris DR, Green JA III: Pyrosequencing study of fecal microflora of autistic and control children. Anaerobe 2010, 16:444-453.

15. Brock M, Buckel W: On the mechanism of action of the antifungal agent propionate. Eur J Biochem 2004, 271:3227-3241.

16. Wajner M, Latini A, Wyse AT, Dutra-Filho CS: The role of oxidative damage in the neuropathology of organic acidurias: insights from animal studies. $J$ Inherit Metab Dis 2004, 27:427-448.

17. Conn AR, Fell DI, Steele RD: Characterization of alpha-keto acid transport across blood-brain barrier in rats. Am J Physiol 1983, 245:E253-E260.

18. Karuri AR, Dobrowsky E, Tannock IF: Selective cellular acidification and toxicity of weak organic acids in an acidic microenvironment. Br J Cancer 1993, 68:1080-1087.

19. Rorig B, Klausa G, Sutor B: Intracellular acidification reduced gap junction coupling between immature rat neocortical pyramidal neurones. J Physiol 1996, 490:31-49.

20. DeCastro M, Nankova BB, Shah P, Patel P, Mally PV, Mishra R, La Gamma EF Short chain fatty acids regulate tyrosine hydroxylase gene expression through a cAMP-dependent signaling pathway. Brain Res Mol Brain Res 2005, 142:28-38.

21. Maurer MH, Canis M, Kuschinsky W, Duelli R: Correlation between local monocarboxylate transporter 1 (MCT1) and glucose transporter 1 (GLUT1) densities in the adult rat brain. Neurosci Lett 2004, 355:105-108.

22. Nakao S, Moriya Y, Furuyama S, Niederman R, Sugiya H: Propionic acid stimulates superoxide generation in human neutrophils. Cell Biol Int 1998, 22:331-337.

23. Hara H, Haga S, Aoyama Y, Kiriyama S: Short-chain fatty acids suppress cholesterol synthesis in rat liver and intestine. J Nutr 1999, 129:942-948,

24. Le Poul E, Loison C, Struyf S, Springael JY, Lannoy V, Decobecq ME, Brezillon S, Dupriez V, Vassart G, Van Damme J, Parmentier M, Detheux M: Functional characterization of human receptors for short chain fatty acids and their role in polymorphonuclear cell activation. J Biol Chem 2003, 278:25481-25489.

25. Parab S, Nankova BB, La Gamma EF: Differential regulation of the tyrosine hydroxylase and enkephalin neuropeptide transmitter genes in rat PC12 cells by short chain fatty acids: concentration-dependent effects on transcription and RNA stability. Brain Res 2007, 1132:42-50.

26. Frye RE, Rossignol DA: Mitochondrial dysfunction can connect the diverse medical symptoms associated with autism spectrum disorders. Pediatr Res 2011, 69:41R-47R.

27. Pastural E, Ritchie S, Lu Y, Jin W, Kavianpour A, Khine Su-Myat K, Heath D, Wood PL, Fisk M, Goodenowe DB: Novel plasma phospholipid biomarkers of autism: Mitochondrial dysfunction as a putative causative mechanism. Prostaglandins Leukot Essent Fatty Acids 2009, 81:253-264.

28. Tamiji J, Crawford DA: The neurobiology of lipid metabolism in autism spectrum disorders. Neurosignals 2010, 18:98-112.

29. Bell JG, MacKinlay EE, Dick JR, MacDonald DJ, Boyle RM, Glen AC: Essential fatty acids and phospholipase A2 in autistic spectrum disorders. Prostaglandins Leukot. Essent Fatty Acids 2004, 71:201-204.

30. Bell JG, Sargent JR, Tocher DR, Dick JR: Red blood cell fatty acid compositions in a patient with autistic spectrum disorder: a characteristic abnormality in neurodevelopmental disorders? Prostaglandins Leukot. Essent Fatty Acids 2000, 63:21-25.

31. Richardson AJ: Clinical trials of fatty acid treatment in ADHD, dyslexia, dyspraxia and the autistic spectrum. Prostaglandins Leukot Essent Fatty Acids 2004, 70:383-390.
32. Vancassel S, Durand G, Barthelemy C, Lejeune B, Martineau J, Guilloteau D, Andres C, Chalon S: Plasma fatty acid levels in autistic children. Prostaglandins Leukot Essent Fatty Acids 2001, 65:1-7.

33. Brugger B, Erben G, Sandhoff R, Wieland FT, Lehmann WD: Quantitative analysis of biological membrane lipids at the low picomole level by nano-electrospray ionization tandem mass spectrometry. Proc Natl Acad Sci U S A 1997, 94:2339-2344.

34. Postle AD, Heeley EL, Wilton DC: A comparison of the molecular species compositions of mammalian lung surfactant phospholipids. Comp Biochem Physiol A Mol Integr Physiol 2001, 129:65-73.

35. Pulfer M, Murphy RC: Electrospray mass spectrometry of phospholipids. Mass Spectrom Rev 2003, 22:332-364.

36. Meguid NA, Atta HM, Gouda AS, Khalil RO: Role of polyunsaturated fatty acids in the management of Egyptian children with autism. Clin Biochem 2008, 41:1044-1048

37. Paxinos G, Watson C: The Rat Brain in Stereotaxic Coordinates. Montreal: Academic Press; 1986.

38. Folch J, Lees M, Sloane Stanley GH: A simple method for the isolation and purification of total lipides from animal tissues. J Biol Chem 1957, 226:497-509.

39. Sherma J, Fried B: Handbook of Thin Layer Chromatography. New York, NY: Marcel and Dekker; 2003:pp. ii-991.

40. Deeley JM, Mitchell TW, Wei X, Korth J, Nealon JR, Blanksby SJ, Truscott RJ: Human lens lipids differ markedly from those of commonly used experimental animals. Biochim Biophys Acta 2008, 1781:288-298.

41. Volkmar FR, Lord C, Bailey A, Schultz RT, Klin A: Autism and pervasive developmental disorders. J Child Psychol Psychiatry 2004, 45:135-170.

42. Cannizzaro C, Monastero R, Vacca M, Martire M: [3 H]-DA release evoked by low $\mathrm{pH}$ medium and internal $\mathrm{H}+$ accumulation in rat hypothalamic synaptosomes: involvement of calcium ions. Neurochem Int 2003, 43:9-17.

43. Remblier C, Pontcharraud R, Tallineau C, Piriou A, Huguet F: Lactic acidinduced increase of extracellular dopamine measured by microdialysis in rat striatum: evidence for glutamatergic and oxidative mechanisms. Brain Res 1999, 837:22-28.

44. Severson CA, Wang W, Pieribone VA, Dohle Cl, Richerson GB: Midbrain serotonergic neurons are central pH chemoreceptors. Nat Neurosci 2003, 6:1139-1140.

45. Bronstein JM, Farber DB, Wasterlain CG: Regulation of type-II calmodulin kinase: functional implications. Brain Res Rev 1993, 18:135-147.

46. Nakao S, Fujii A, Niederman R: Alteration of cytoplasmic Ca2+ in resting and stimulated human neutrophils by short-chain carboxylic acids at neutral pH. Infect Immun 1992, 60:5307-5311.

47. Fukuchi M, Nii T, Ishimaru N, Minamino A, Hara D, Takasaki I, Tabuchi A, Tsuda M: Valproic acid induces up- or down-regulation of gene expression responsible for the neuronal excitation and inhibition in rat cortical neurons through its epigenetic actions. Neurosci Res 2009, 65:35-43.

48. Bayir $H$, Tyurin VA, Tyurina $Y Y$, Viner $R$, Ritov V, Amoscato AA, Zhao $Q$, Zhang XJ, Janesko-Feldman KL, Alexander H, Basova LV, Clark RS, Kochanek PM, Kagan VE: Selective early cardiolipin peroxidation after traumatic brain injury: an oxidative lipidomics analysis. Ann Neurol 2007, 62:154-169.

49. Little SJ, Lynch MA, Manku M, Nicolaou A: Docosahexaenoic acid-induced changes in phospholipids in cortex of young and aged rats: a lipidomic analysis. Prostaglandins Leukot Essent Fatty Acids 2007, 77:155-162.

50. Hon GM, Hassan MS, van Rensburg SJ, Abel S, van JP, Erasmus RT, Matsha T: Red blood cell membrane fluidity in the etiology of multiple sclerosis. J Membr Biol 2009, 232:25-34.

51. Kakela R, Somerharju P, Tyynela J: Analysis of phospholipid molecular species in brains from patients with infantile and juvenile neuronalceroid lipofuscinosis using liquid chromatography-electrospray ionization mass spectrometry. J Neurochem 2003, 84:1051-1065.

52. Bu B, Ashwood P, Harvey D, King IB, Water JV, Jin LW: Fatty acid compositions of red blood cell phospholipids in children with autism. Prostaglandins Leukot Essent Fatty Acids 2006, 74:215-221.

53. Sliwinski S, Croonenberghs J, Christophe A, Deboutte D, Maes M: Polyunsaturated fatty acids: do they have a role in the pathophysiology of autism? Neuro Endocrinol Lett 2006, 27:465-471.

54. Farooqui AA, Ong WY, Horrocks LA: Biochemical aspects of neurodegeneration in human brain: involvement of neural membrane 
phospholipids and phospholipases A2. Neurochem Res 2004, 29: 1961-1977.

55. Chauhan A, Chauhan V: Oxidative stress in autism. Pathophysiology 2006, 13:171-181.

56. Chauhan A, Chauhan V, Brown WT, Cohen I: Oxidative stress in autism: increased lipid peroxidation and reduced serum levels of ceruloplasmin and transferrin-the antioxidant proteins. Life Sci 2004, 75:2539-2549.

57. Chauhan V, Chauhan A, Cohen IL, Brown WT, Sheikh A: Alteration in amino-glycerophospholipids levels in the plasma of children with autism: a potential biochemical diagnostic marker. Life Sci 2004, 74: 1635-1643.

58. Vargas DL, Nascimbene C, Krishnan C, Zimmerman AW, Pardo CA: Neuroglial activation and neuroinflammation in the brain of patients with autism. Ann Neurol 2004, 57:67-81.

59. Farooqui AA, Horrocks LA: Plasmalogens, phospholipase A(2), and docosahexaenoic acid turnover in brain tissue. J Mol Neurosci 2001, 16:263-272.

60. Allen HG, Allen JC, Boyd LC, ston-Mills BP, Fenner GP: Determination of membrane lipid differences in insulin resistant diabetes mellitus type 2 in whites and blacks. Nutrition 2006, 22:1096-1102.

61. Schuchardt JP, Huss M, Stauss-Grabo M, Hahn A: Significance of long-chain polyunsaturated fatty acids (PUFAs) for the development and behaviour of children. Eur J Pediatr 2009, 169:149-164.

62. Gorgas K, Teigler A, Komljenovic D, Just WW: The ether lipid-deficient mouse: tracking down plasmalogen functions. Biochim Biophys Acta 2006, 1763:1511-1526

63. Dommels YE, Alink GM, Linssen JP, van OB: Effects of $n-6$ and $n-3$ polyunsaturated fatty acids on gap junctional intercellular communication during spontaneous differentiation of the human colon adenocarcinoma cell line Caco-2. Nutr Cancer 2002, 42:125-130.

64. Martinez AD, Saez JC: Regulation of astrocyte gap junctions by hypoxiareoxygenation. Brain Res Brain Res Rev 2000, 32:250-258.

65. Aylsworth CF, Trosko JE, Welsch CW: Influence of lipids on gap-junctionmediated intercellular communication between Chinese hamster cells in vitro. Cancer Res 1986, 46:4527-4533.

66. Champeil-Potokar G, Chaumontet C, Guesnet P, Lavialle M, Denis I: Docosahexaenoic acid (22:6n-3) enrichment of membrane phospholipids increases gap junction coupling capacity in cultured astrocytes. Eur J Neurosci 2006, 24:3084-3090.

67. Rouach N, Avignone E, Meme W, Koulakoff A, Venance L, Blomstrand F, Giaume C: Gap junctions and connexin expression in the normal and pathological central nervous system. Biol Cell 2002, 94:457-475.

doi:10.1186/1742-2094-9-153

Cite this article as: Thomas et al: The enteric bacterial metabolite propionic acid alters brain and plasma phospholipid molecular species: further development of a rodent model of autism spectrum disorders. Journal of Neuroinflammation 2012 9:153.

\section{Submit your next manuscript to BioMed Central and take full advantage of:}

- Convenient online submission

- Thorough peer review

- No space constraints or color figure charges

- Immediate publication on acceptance

- Inclusion in PubMed, CAS, Scopus and Google Scholar

- Research which is freely available for redistribution 